\section{Adding calcitriol to docetaxel results in a valuable survival benefit}

Docetaxel-containing chemotherapy is the standard treatment for patients with metastatic, androgen-independent prostate cancer (AIPC). Vitamin D receptor ligands such as calcitriol have antiproliferative, proapoptotic and antiangiogenic effects that complement the cytotoxic effects of docetaxel; however, calcitriol's antineoplastic effects require intermittent, supraphysiologic dosing. Beer and colleagues, therefore, compared the safety and efficacy of high-dose oral calcitriol (DN-101) and docetaxel with that of placebo and docetaxel.

In this multicenter, double-blind trial, 250 adults with AIPC were randomly allocated to receive docetaxel plus either placebo or DN-101. Patients received $36 \mathrm{mg} / \mathrm{m}^{2}$ body surface area intravenous docetaxel weekly for 3 weeks (first week only $27 \mathrm{mg} / \mathrm{m}^{2}$ ) on a 4 -week cycle. One day before docetaxel administration, patients received $45 \mu \mathrm{g}$ DN-101 or placebo orally. Patients also received dexamethasone before and after docetaxel infusions. Treatment continued until progression or unacceptable toxicity occurred.

At the time of analysis (median follow-up 18.3 months), $92 \%$ of the patients had completed treatment and $49 \%$ had died. The study end point (a confirmed PSA decline of $\geq 50 \%$ within 6 months of enrollment) was reached by similar proportions in both treatment groups (49\% placebo, 58\% DN-101; overall response rates $52 \%$ and $63 \%$, respectively). Median survival for placebo-treated patients was 16.4 months, compared with an estimated 24.5 months for DN101-treated patients. DN-101 did not increase the toxicity of docetaxel-containing chemotherapy.

The authors suggest that adding calcitriol to docetaxel resulted in a valuable survival benefit; however, this result needs further validation.

\footnotetext{
Original article Beer TM et al. (2007) Double-blinded randomized study of high-dose calcitriol plus docetaxel compared with placebo plus docetaxel in androgenindependent prostate cancer: a report from the ASCENT investigators. J Clin Oncol 25: 669-674
}

\section{Risk of death from prostate cancer increases as time to androgen suppression increases}

Androgen deprivation therapy (ADT) is administered to treat PSA recurrence following surgery or radiotherapy for prostate cancer. Men who achieve undetectable $(<0.2 \mathrm{ng} / \mathrm{ml})$ PSA levels within 8 months of ADT have improved survival. D'Amico and colleagues, therefore, conducted a retrospective study to investigate whether the time to achieve an undetectable PSA level influenced the time to prostate-cancer-specific mortality.

The analysis included 585 men (median age 69 years) who received ADT to treat rising PSA levels after radical prostatectomy $(n=415)$ or radiation therapy ( $n=170)$, and who subsequently achieved undetectable PSA levels.

In total, 23 patients died; 4 deaths were caused by prostate cancer. The median time to achieve undetectable PSA levels was 4.6 months (range 2.8-7.8 months). After adjustment for known prognostic factors, a long time to reach undetectable PSA levels, a short PSA doubling time before introduction of ADT, and high-grade prostate cancer were all associated with early prostate-cancer-specific mortality. Those men who took longer than the median time to achieve undetectable PSA levels also had shorter times to death from all causes. Men whose PSA became undetectable within 4.6 months had an estimated 5-year prostatecancer-specific mortality of $12 \%$ and 5 -year all-cause mortality of $0 \%$-compared with $23 \%$ and $7 \%$, respectively, among those who took longer than 4.6 months.

The authors suggest that men who take a long time to achieve undetectable PSA levels after the introduction of ADT should be considered for enrollment into randomized trials of immediate versus delayed chemotherapy.

Original article D'Amico AV et al. (2007) Time to an undetectable prostate-specific antigen (PSA) after androgen suppression therapy for postoperative or postradiation PSA recurrence and prostate cancer-specific mortality. Cancer 109: $1290-1295$

\section{New method for measuring serum PSA level might enable mass screening programs}

The standard technique for measurement of serum PSA levels, although accurate and reliable, requires expensive equipment and skilled medical personnel, which prohibits mass screening. Azzouzi and colleagues have evaluated the reliability of a new method of PSA measurement (called the nanotest), with which 